\title{
Management of abnormal uterine bleeding by northern, rural and isolated primary care physicians: PART II: What do we need? Simone N Vigod ${ }^{1}$ and Donna E Stewart*2
}

Address: ${ }^{1}$ Faculty of Medicine, University of Toronto, Toronto Canada and ${ }^{2}$ Professor and Chair of Women's Health, University Health Network, 657 University Ave., ML 2-004, Toronto, Ontario, M5G 2N2, Canada

E-mail: Simone N Vigod - simone.vigod@utoronto.ca; Donna E Stewart* - donna.stewart@uhn.on.ca

*Corresponding author

Published: 20 November 2002

BMC Women's Health 2002, 2:II

This article is available from: http://www.biomedcentral.com/I472-6874/2/II

(C) 2002 Vigod and Stewart; licensee BioMed Central Ltd. This is an Open Access article: verbatim copying and redistribution of this article are permitted in all media for any purpose, provided this notice is preserved along with the article's original URL.
Received: 20 June 2002

Accepted: 20 November 2002

\begin{abstract}
Background: Abnormal uterine bleeding (AUB) is a common problem that affects one in five women during the pre-menopausal years. It is frequently managed by family physicians, especially in northern, rural and isolated areas where severe shortages of gynecologists exist.

Methods: We surveyed 194 family physicians in northern, rural and isolated areas of Ontario, Canada to determine their educational and resource needs for the management of AUB, with a specific focus on the relevance and feasibility of using clinical practice guidelines (CPGs).

Results: Most physicians surveyed did not use CPGs for the management of AUB because they did not know that such guidelines existed. The majority were interested in further education on the management of AUB through mailed CPGs and locally held training courses. A major theme among respondents was the need for more timely and effective gynecological referrals.
\end{abstract}

Conclusion: A one-page diagnostic and treatment algorithm for AUB would be easy to use and would place minimal restrictions on physician autonomy. As the majority of physicians had Internet access, we recommend emailing and web posting in addition to mailing this algorithm. Local, handson courses including options for endometrial biopsy training would also be helpful for northern, rural and isolated physicians, many of whom cannot readily take time away from their practices.

\section{Background}

Abnormal uterine bleeding (AUB) is a common problem that affects one in five women during the pre-menopausal years [1]. The bleeding may be unmanageable, disruptive to daily activities and damaging to self-esteem and personal relationships. Primary care physicians (family doctors) are often responsible for the management of women with heavy bleeding, especially in northern, rural and isolated areas where severe shortages of gynecologists exist. It is vital to ensure that these physicians have the tools to provide optimal management. This study addresses the educational and resource needs of family physicians in northern, rural and isolated areas of Ontario, Canada with respect to the management of AUB with a specific focus on the relevance and feasibility of using clinical practice guidelines (CPGs).

\section{Methods}

We developed a five-page questionnaire entitled "Management of Heavy Vaginal Bleeding: Northern and Rural Primary Care Physicians" composed of four parts, of which parts: I. Physician Information, III. Clinical Practice 
Table I: Physicians reasons for not using a CPG for AUB or hysterectomy $(\mathrm{N}=$ I77)

\begin{tabular}{lc}
\hline Deterrent & N (\%) \\
\hline & $112(63.3)$ \\
I did not know that such a guideline existed & $35(19.8)$ \\
I am not familiar enough with the guideline & $26(14.7)$ \\
I am confident in managing abnormal uterine bleeding without a guideline & $9(5.1)$ \\
I don't have time to look through the guideline & $6(3.4)$ \\
I have searched, but was unable to find such a guideline & $2(1.1)$ \\
I never see cases of abnormal uterine bleeding & $1(0.6)$ \\
I do not agree with the guideline(s) & I (0.6) \\
I do not believe in CPGs in general & \\
\hline
\end{tabular}

Guideline Use and IV. Education and Resource Needs Assessment, are relevant to this paper (see accompanying article "Management of Abnormal Uterine Bleeding by Northern, Rural and Isolated Primary Care Physicians: Part I: How Are We Doing?" for survey instrument - Appendix 1). We collected information on physicians' sex, age, history of clinical practice, Internet access (for availability of information), previous obstetrical experience (possible indication of comfort level with managing gynecological problems) and Canadian College of Family Physicians (CCFP) membership status (access to continuing medical education resources). Questions on CPG use were developed on the basis of a literature review in June, 2000 on clinical practice guideline dissemination and adherence. Medline keywords were clinical practice guideline, adherence and family physician [2-8]. The education and resource needs assessment consisted of both "mark your choices" and open-ended questions.

A package including a cover letter, stamped return envelope and the questionnaire was mailed to 676 physicians in Northern Ontario. Reminder letters and replacement questionnaires were sent 3 and 7 weeks respectively after the original, using a modification of the Dillman (TDM) approach [9]. Due to incorrect address or specialty information (i.e. respondents were not family physicians), fifty-four surveys were returned. From the remaining 622 potential participants, there were 194 completed responses (Response Rate $=31 \%$ ).

The Statistical Package for the Social Sciences (SPSS, version 10) was used for statistical analysis. Descriptive analyses of all continuous and categorical variables were conducted. Chi square tests of independence, independent sample t-tests and analyses of variance (ANOVA) investigated whether responses were dependent on physician variables.

\section{Results \\ Physician information}

Detailed physician information has been reported in the accompanying article. Significantly, the average age of the responding physicians was 43 years, with an average of 15 years in family practice. In accordance with the gender distribution of physicians in Ontario, $37.5 \%$ of the respondents were female, $62.5 \%$ were male, with males being significantly older and with more years in family practice than females. All physicians practiced in Northern Ontario, with $45.1 \%$ also designating their practice population as "rural" and $17.5 \%$ as "isolated". These categories were not mutually exclusive (i.e. a physician could practice in an area that is rural and isolated). Half of the respondents were practicing in areas with populations of less than 15000 people, with the remainder in larger towns $(25 \%)$ or small cities $(25 \%)$. Three quarters $(75 \%)$ were members of the Canadian College of Family Physicians (CCFP) with a higher proportion of women being members than men. Almost all (92.7\%) of respondents had Internet access either in their homes or offices.

\section{Clinical practice guidelines}

Most physicians (91.2\%) did not use a CPG for the management of AUB. Reasons included not knowing that such a guideline existed (63.3\%), not being familiar enough with it to use it $(19.8 \%)$ and feeling confident managing AUB without a CPG (14.7\%) (Table 1). There were physicians who were "somewhat" (13.0\%), "fairly" (10.4\%) or "quite/very" (2.0\%) familiar with a guideline for the management of AUB but chose not to use it. They thought that the guideline would "require a change in practice habits", "restrict their autonomy", was "not applicable to their practice" or was "not consistent with their clinical experience". Guideline sources were continuing medical education (CME) programs (47.5\%), journals (36.2\%) and medical school/residency training (35.0\%). Only $8.6 \%$ cited the Internet as their source. The most popular guideline used was from the Society of Obstetricians and Gynecologists of Canada (SOGC) $(\mathrm{N}=7)$, with others using 
Table 2: Continuing medical education: desired format $(N=179)$

\begin{tabular}{lc}
\hline Format & $\mathbf{N}(\%)$ \\
\hline & \\
Copies of a clinical practice guideline & 14I (78.8) \\
Tips for medical/hormonal management of AUB & $90(50.3)$ \\
Journal articles & $69(38.5)$ \\
Courses held locally & $63(35.2)$ \\
Pamphlets & $38(21.2)$ \\
Web-based & $36(20.1)$ \\
Hands-on instruction (e.g. endometrial sampling) & $33(18.4)$ \\
Videotapes & $27(15.1)$ \\
Audiotapes & I7 (9.5) \\
Telemedicine & $17(9.5)$ \\
Calendar charts for quantitative measurement of bleeding & I7 (9.5) \\
Courses held at a medical school & I5 (8.4)
\end{tabular}

American College of Obstetricians and Gynecologists, textbooks or unspecified resources.

Seventeen responding physicians $(8.8 \%)$ used a CPG for AUB or hysterectomy in their practices, citing "relevance to practice" (92.9\%), "easy to use" (85.7\%), "evidencebased" (78.6\%) and "consistent with clinical experience" $(78.6 \%)$ as the greatest incentives for use.

\section{Education and resource needs}

Almost all physicians (92.7\%) were interested in receiving continuing medical education on the management of AUB, with popular formats being mailed copies of a CPG, tips for medical/hormonal management of AUB, journal articles and courses held locally (Table 2). Several physicians suggested training in in-office endometrial biopsies.

Increasing access to gynecologists was a common suggestion for improving management of AUB. Family physicians reported that the average length of time that a woman in their practices such as the one in our clinical scenario would wait for a gynecological consult was $7.1+$ / - 4.7 weeks (range 1-28). Less than half $(45.4 \%)$ of the physicians could refer such patients to gynecologists in their hometowns and $42.3 \%$ of physicians' reported that patients would have to commute between 1 and 3 hours to see a gynecologist while $8.8 \%$ reported commutes of over three hours. Physicians were also concerned with the "quality of the gynecological referral", wanting "more and better gynecologists to work locally", more "visiting specialist programs" and "easier referral with more informative consultant reports".

\section{Discussion}

The moderate response rate to this survey limits the generalizability of the results as we discuss in the accompany- ing article. Although the creation of strategies for improving the management of AUB will be based on the responses to this questionnaire, it is hoped that the development of convenient, easy-to-use educational tools and resources will also appeal to those family physicians who did not respond to the survey.

It appears that most family physicians did not use CPGs for the management of AUB because they did not know of such guidelines. These guidelines are published by specialist (Obstetrics and Gynecology) organizations [1013]. It is unreasonable to expect primary care physicians to peruse all specialty journals that could have some relevance to their practice. However, due to the prevalence of AUB, there is a need for a mass awareness campaign for physicians on where to find information on the management of AUB. Clearly, the internet is an untapped resource as most of the existing CPGs are available online. However, many physicians suggested mailed clinical practice guidelines and some physicians urgently requested that the researchers mail them a copy of a CPG for AUB. Therefore, mailings (by physician organizations with accurate lists of northern family physicians and their web addresses) may be a useful option for practitioners who do not access guidelines electronically or who prefer hard copies of guidelines.

Another vital question is whether physicians would use guidelines if they were more readily available. Respondents who commented on the content of current AUB guidelines cited incentives and barriers to guideline use that are consistent with the findings in the general literature on guideline use-relevance, ease of use, consistency with their clinical experience and a lack of restriction to their autonomy [2-8]. With this in mind, we recommend a one page mailing, emailing and web posting of the sali- 
ent management points for AUB including a diagnostic approach with specific treatment options. This would be easy to use, would place minimal restrictions on physician autonomy and could be easily derived from existing CPGs on AUB. Once physicians become more familiar with a CPG for AUB management, specific incentives and barriers to guideline use for AUB by family physicians can be explored more fully.

The recurrent theme of more timely and effective gynecological referrals is a difficult issue. There is no immediate way to increase the number of gynecologists in these regions. Augmentation of visiting specialist programs would be difficult in an already understaffed specialty. The development of a triage system for gynecological referral is not an imminent possibility. For now, it appears that primary care physicians, who are also in short supply in many northern areas, must become more comfortable managing abnormal bleeding while waiting for, or instead of, a gynecological consultation. As several physicians suggested, clinical practice guidelines and locally held training courses would be an appropriate start.

\section{Conclusion}

The needs outlined in this study should be considered for their applicability when developing educational programs and materials on abnormal uterine bleeding for family physicians and patients in northern, rural and isolated communities. We recommend that a one page summary of the necessary investigations, treatment options and their costs be mailed and also be made available online (with the CPG web address on an adhesive label in the mailed package). Educational initiatives for physicians and patients are also necessary and should include local, hands-on courses (including options for endometrial biopsy training) where requested by physicians, most of whom cannot readily take time away from their practices.

\section{Competing interests}

Competing interests: None declared.

\section{Authors' contributions}

Dr. Stewart conceived of the project, supervised the work, and fine-tuned the write-up.

Ms. Vigod conducted the literature review, developed the questionnaire, conducted the survey, analyzed the data and drafted the report.

\section{Acknowledgments}

We are grateful to Dr NA Leyland for his suggestions on this project and manuscript.

\section{References}

I. London R, Holzman M, Rubin D, Moffitt B: Payer cost savings with endometrial ablation therapy. Am J Manag Care I999, 5:889-897
2. Bell N: Implementation of Clinical Practice Guidelines into Practice: Future Strategies. The Guideline 1999

3. Broder MS, Kanouse DE, Mittman BS, Bernstein SJ: The appropriateness of recommendations for hysterectomy. Obstet Gynecol 2000, 95: 199-205

4. Cabana MD, Rand CS, Powe NR, Wu AW, Wilson MH, Aboud PAC, Rubin HR: Why don't physicians follow clinical practice guidelines? A framework for improvement. JAMA 1999, 282:14581465

5. Feder G, Eccles M, Grol R, Griffiths C, Grimshaw J: Using clinical guidelines. BMJ 1999, 3 I 8:728-730 [http://www.bmj.com/cgi/content/full/3|8/7|85/728]

6. Grol R, Dalhuijsen J, Thomas S, Veld C, Rutten G, Mokkink H: Attributes of clinical guidelines that influence use of guidelines in general practice: observational study. BMJ 1998, 317:858861

7. Iscoe N: "We may get this horse to water, but will it drink?" CMAJ 1998, I 58:345-346

8. Hayward RS, Guyatt GH, Moore KA, McKibbon KA, Carter AO: Canadian physician's attitudes about and preferences regarding clinical practice guidelines. CMAJ 1997, 156:17/5-23

9. Dillman DA: Mail and Internet surveys: The tailored method. New York: John Wiley and Sons 1999

10. Society of Obstetricians and Gynecologists of Canada: Clinical practice guidelines for hysterectomy (policy statement). J Soc $O b-$ stet Gynecol Can 1996, 47:

II. Vilos GA, Lefebvre G, Graves GR: Guidelines for the management of abnormal uterine bleeding. SOGC Clinical Practice Guidelines 2001, 106:

12. ACOG: Technical Bulletin: Dysfunction uterine bleeding. Washington: American College of Obstetricians and Gynecologists 1989

13. ACOG: Quality assessment and improvement in obstetrics and gynecology: ACOG criteria sets. Washington: American College of Obstetricians and Gynecologists 1994

\section{Pre-publication history}

The pre-publication history for this paper can be accessed here:

http://www.biomedcentral.com/1472-6874/2/11/prepub

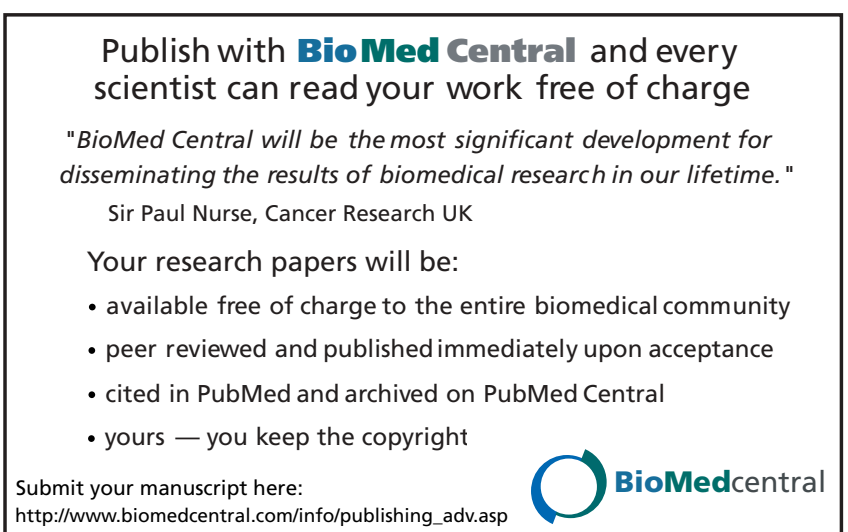

